CYBERNETICS AND INFORMATION TECHNOLOGIES • Volume 16, No 6

Special issue with selection of extended papers from 6th International Conference on Logistic, Informatics and Service Science LISS'2016

Sofia • 2016

\title{
Analysis of Various ESDD of Contaminant Insulator Flashover Acoustic Signal by Wavelet Packet
}

\author{
Hong Kun Wang ${ }^{1,2}$, Shou Xiang Wang ${ }^{1}$, Jing Nie ${ }^{2}$ \\ ${ }^{1}$ School of Electrical Engineering and Automation, Tianjin University, Tianjin 300072, China \\ ${ }^{2}$ The Mechanical and Electrical Institute, Shihezi University, Shihezi 832003, China \\ Emails:whkmyr@163.com sxwang@tju.edu.cn niejing19@163.com
}

\begin{abstract}
With the formation of China's large power grid, the security of the network is particularly important. The contaminant flashover of insulators has a serious impact on the operation safety of a high voltage power network. In this paper, the acoustic signals' characteristics of the contaminant insulators flashover are analyzed, and, as a result, the correlation between the acoustic signal and the contaminant insulator flashover is established. To experiment with contaminant insulator for three different Equivalent Salt Deposit Densities (ESDD), acoustic signals were collected separately. Then, the contaminant insulators' acoustic signals of flashover were analyzed by wavelet packet. The characteristics of the signals were obtained, and they can be judged for contaminant flashover warning.
\end{abstract}

Keywords: Contaminant insulator flashover, acoustic signal, Equivalent Salt Deposit Density (ESDD), wavelet packet.

\section{Introduction}

With the continuous improvement in the level of capacity of China's power grid and increasingly serious environmental pollution, insulator flashover accidents have occurred, seriously affecting the safety and stable operation of the power grid [1-5]. In recent years, great changes in China's energy distribution have taken place. Power transmission capacity has been improved after the west-east power transmission project, so that the voltage level of the power grid is upgrading continuously. A large nationwide network has been formed, and the safe operation of the power grid is becoming increasingly important. The secure operation of the 
power grid is badly threatened by contaminant flashover of insulators. According to the statistics, the accidents caused by contaminant flashover are ranked on the second position, only behind lightning. As the success rate of automatic reclosing of pollution flashover is very low, the loss is ten times more than that of lightning. So it is essential for the reliable performance of the large networks to predict contaminant insulators flashover accurately, as well as to take measures to deal with it.

Polluted insulators' discharge comes with acoustic emission [6-10], and measuring the acoustic signal can be used to detect insulator external insulation condition, in order to reflect the external insulation condition of the insulator more accurately. This is particularly important for recognizing its contaminant flashover. Selection of contaminant flashover acoustic emission signal parameters for discharge recognition plays an important role, and can affect the recognition directly.

The method to prejudge contaminant flashover in China at the present stage is usually to measure insulator's leakage currents, but the leakage current is a direct measurement that needs special insulators, or technical renovation, so it is not good for large-scale use on insulators. With the development of advanced computer monitoring technology, the new monitoring methods have been optimized, such as ultrasonic testing, ultraviolet pulse detection and laser detection. In the discharge process of insulator flashover, there is a discharge acoustic signal, which could be from weak to strong; that means the contaminant flashover acoustic signal can better characterize the intensity of contamination insulator discharge [11-16]. Therefore, by detecting insulator flashover discharge signals, an insulator flashover warning can be achieved according to the flash discharge characteristics of the acoustic signal at different stages.

In order to realize the warning of acoustic signals of the contaminant flashover, the analysis on the characteristics of discharge acoustic signals is essential. Wavelet packet analysis is a complete analysis process of the signal, where all the features of the whole signal can be reflected. In this paper, the acoustic signals of insulators are reconstructed by the wavelet packet decomposition, and the insulator pollution flashover discharge warning is realized according to the characteristics of the reconstructed signals.

\section{Acquisition of sound signals in contaminant insulator flashover}

\subsection{Principle of detection of acoustic signal from contaminant insulator flashover}

Equivalent Salt Deposit Density (ESDD) has been widely used in the contamination testing process, in the mechanism of analysis of pollution flashover, and in the amount of contaminant on the power system. The contaminant insulator flashover is a process of releasing energy, and part of the acoustic signal is the release of energy. In [7] is pointed out that the square root of flashover energy and acoustic signal amplitude has a positive relationship. The whole process of acoustic 
signaling throughout the contaminant insulator flashover, namely from the initial corona discharge to the partial discharge, has been developed.

\subsection{Test design}

In order to better study acoustic signals in contaminant insulator flashover, tests on contaminant insulator flashover were carried out in the artificial mist chamber on the level of high voltage, and acoustic signals were collected. CQSB 6/50 experimental models of high voltage test transformers produced by Wuhan Electric Power Equipment Co., Ltd as well as the XP-70 pin type porcelain insulators were used in the tests. The monitoring devices consist of an acoustic sensor, an amplifier circuit, filter circuit, data acquisition circuit, signal processing circuit, and communication circuit. The frequency of the $40 \mathrm{kHz}$ ultrasonic sensor was selected because the flashover discharge energy acoustic signals are mainly in $10-50 \mathrm{kHz}$. Fig. 1 shows the diagram of wiring principle.

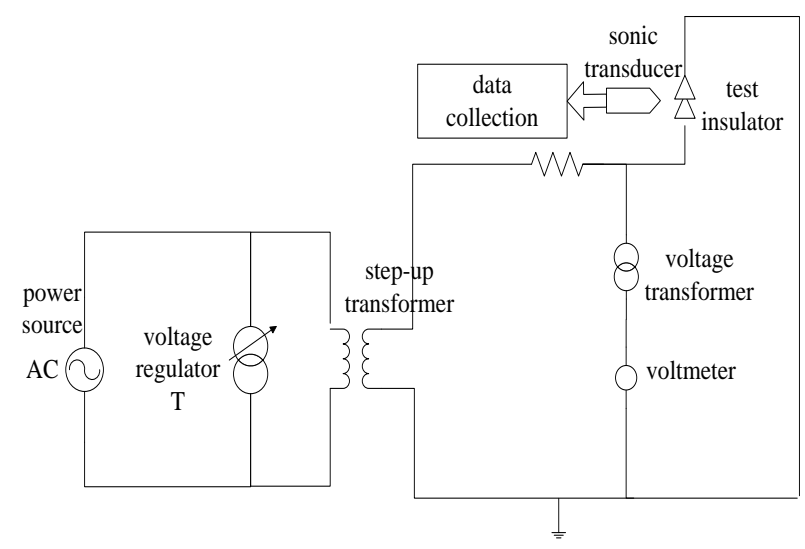

Fig. 1. Test wiring principle

Insulators with different materials and salt densities have the same trend on contaminant insulator flashover of acoustic signals using a set of experimental data, with the test design as follows: according to one kind of moderate pollution level with the IEC standard, ESDD are taken as $\rho_{\text {ESDD }}=0.03 \mathrm{mg} / \mathrm{cm}^{2} ; \rho_{\mathrm{ESDD}}=0.1 \mathrm{mg} / \mathrm{cm}^{2}$; $\rho_{\mathrm{ESDD}}=0.2 \mathrm{mg} / \mathrm{cm}^{2}$. The cleaning spray method is used for the insulator string XP-70 contaminant insulator flashover test. Polluted insulator string in the filth of the saturated humidity discharge acoustic signal is collected under three different equivalent salt densities. Through real-time monitoring of the temperature and humidity monitoring system, stabilizing the relative humidity is always in a saturated state. In order to detect the acoustic signals of polluted insulators in saturated humidity, the samples of polluted insulators with different ESDDs were placed in a fog chamber $20 \mathrm{~min}$, after the fouling layer of the insulators surface were wet. With waste in this condition, three groups of different degrees of polluted insulator were selected, and the polluted insulator acoustic signals were recorded within $20 \mathrm{~min}$, resulting in the average of the three groups of samples as the final test data. The acoustic signal of XP-70 under the insulator experiments on different 
degrees of pollution is shown in Table 1. The test data reveals that the insulator polluted by different ESDDs and the contamination flashover acoustic emission signals have the same trend; namely, the more contamination from the insulator, the stronger the signal is. This point is similar as described in literature [10-14].

Table 1. Relation between average of acoustic signal and ESDD of XP-70

\begin{tabular}{|l|c|c|c|c|c|c|}
\hline NO of group & $\begin{array}{c}\text { ESDD } \\
\left(\mathrm{mg} / \mathrm{cm}^{2}\right)\end{array}$ & $\begin{array}{c}\text { Acoustic } \\
\text { signal } \\
\text { average } \\
(\mathrm{mV})\end{array}$ & $\begin{array}{c}\text { ESDD } \\
\left(\mathrm{mg} / \mathrm{cm}^{2}\right)\end{array}$ & $\begin{array}{c}\text { Acoustic } \\
\text { signal } \\
\text { average } \\
(\mathrm{mV})\end{array}$ & $\begin{array}{c}\mathrm{ESDD} \\
\left(\mathrm{mg} / \mathrm{cm}^{2}\right)\end{array}$ & $\begin{array}{c}\text { Acoustic } \\
\text { signal } \\
\text { average } \\
(\mathrm{mV})\end{array}$ \\
\hline First group & 0.03 & 0.13 & 0.1 & 0.38 & 0.2 & 0.94 \\
\hline Second group & 0.03 & 0.12 & 0.1 & 0.42 & 0.2 & 0.96 \\
\hline Third group & 0.03 & 0.12 & 0.1 & 0.39 & 0.2 & 0.93 \\
\hline
\end{tabular}

2.3. Acoustic signals acquisition and spectrum processing

In order to better collect acoustic signals of contaminant insulator flashover, the acoustic sensor is installed close to the insulator artificial fog chamber inside. This is reducing the interference of external sound, attenuation compensation and the filter circuit are used in the signal processing circuit. The polluted insulator in the test case was: $\rho_{\mathrm{ESDD}}=0.1 \mathrm{mg} / \mathrm{cm}^{2} ; \rho_{\mathrm{ESDD}}=0.1 \mathrm{mg} / \mathrm{cm}^{2} ; \rho_{\mathrm{ESDD}}=0.2 \mathrm{mg} / \mathrm{cm}^{2}$. The contaminant insulator flashover discharge at the acoustic signal waveform under different ESDDs is shown on Fig. 2.

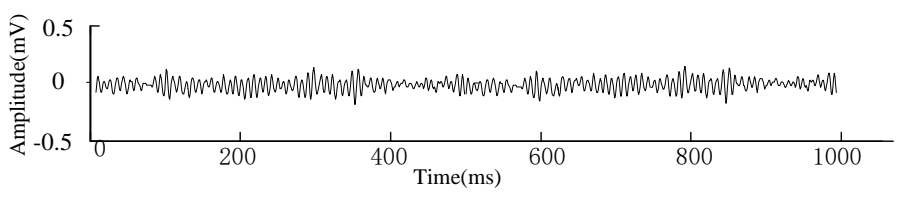

(a) Waveform of acoustic signal $\rho_{\mathrm{ESDD}}=0.03 \mathrm{mg} / \mathrm{cm}^{2}$

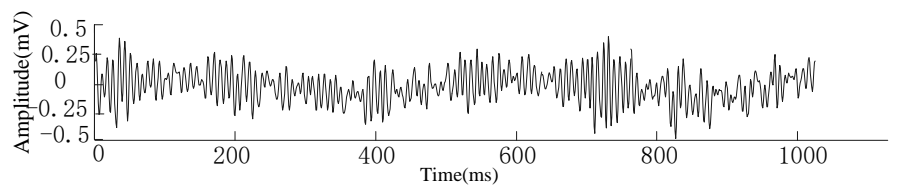

(b) Waveform of acoustic signal $\rho_{\mathrm{ESDD}}=0.1 \mathrm{mg} / \mathrm{cm}^{2}$

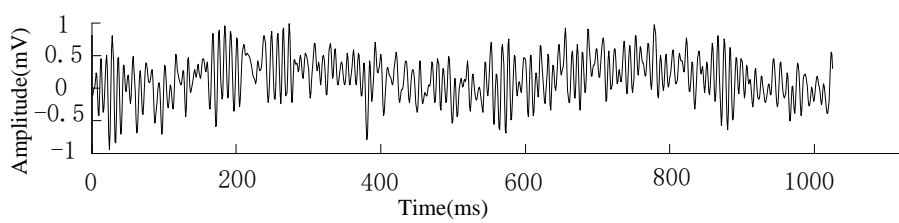

(c) Waveform of acoustic signal $\rho_{\mathrm{ESDD}}=0.2 \mathrm{mg} / \mathrm{cm}^{2}$

Fig. 2. Waveforms of insulator pollution flashover discharge acoustic signal under different ESDDs 
2.4. Characteristics of the acoustic signal in contaminant insulator flashover

Under different equivalent salt deposit densities $\rho_{\mathrm{ESDD}}=0.03 \mathrm{mg} / \mathrm{cm}^{2}$; $\rho_{\text {ESDD }}=0.1 \mathrm{mg} / \mathrm{cm}^{2} ; \rho_{\mathrm{ESDD}}=0.2 \mathrm{mg} / \mathrm{cm}^{2}$ the acoustic signal can well reflect similar characteristics. The discharge characteristics of the acoustic signal and spectrum are shown on Fig. 3.

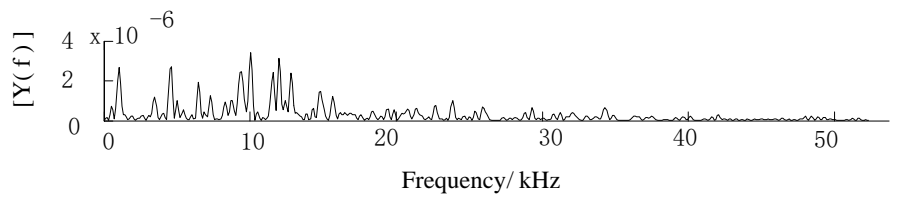

(a) Spectrum diagram of acoustic signal $\rho_{\mathrm{ESDD}}=0.03 \mathrm{mg} / \mathrm{cm}^{2}$

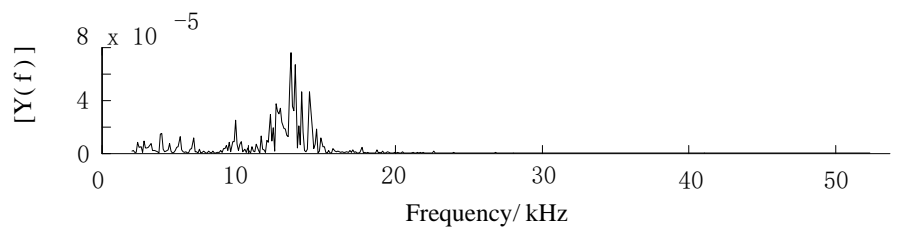

(b) Spectrum diagram of acoustic signal $\rho_{\mathrm{ESDD}}=0.1 \mathrm{mg} / \mathrm{cm}^{2}$

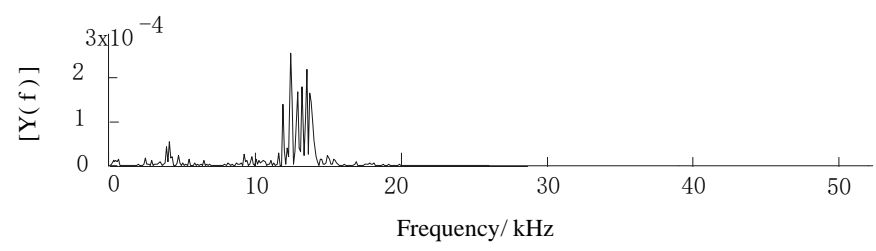

(c) Spectrum diagram of acoustic signal $\rho_{\mathrm{ESDD}}=0.2 \mathrm{mg} / \mathrm{cm}^{2}$

Fig. 3. Spectrum diagrams of pollution flashover discharge under different ESDDs of acoustic signal

Throughout the process of contaminant insulator flashover under different ESDDs, the acoustic signal remains from the beginning to the end during the discharge. The acoustic signal is coherent, sometimes strong and sometimes weak, but the trend of the development of the acoustic signal is the same. Analysis of the discharge acoustic signal reveals that the discharge acoustic signal at the equivalent salt deposit density $\rho_{\text {ESDD }}=0.03 \mathrm{mg} / \mathrm{cm}^{2}$ is weak, and the frequency focused on $2-15 \mathrm{kHz}$, but the spectral energy is low, as is shown in Fig. 3a. In equivalent salt deposit density $\rho_{\mathrm{ESDD}}=0.1 \mathrm{mg} / \mathrm{cm}^{2}$ the discharge phase amplitude and spectral energy of the acoustic signal changes significantly. The frequency at $12 \mathrm{kHz}$ with glaring pulse is shown in Fig. 3b. In equivalent salt deposit density $\rho_{\text {ESDD }}=0.2 \mathrm{mg} / \mathrm{cm}^{2}$ discharge phase amplitude and spectral energy acoustic signal reached the maximum. The frequency between $12-14 \mathrm{kHz}$ continuous pulses is shown on Fig. 3c.

The flashover discharge acoustic signal has the following characteristics:

1) Different ESDD pollution flashover discharge acoustic signal spectrum ranges from 2 up to $15 \mathrm{kHz}$. 
2) Flashover discharge sound frequency, amplitude and flashover risk rating are as positive as the pollution level. warning.

3) The change in frequency and amplitude can be used for pollution flashover

\section{Acoustic signal analysis of insulator based on wavelet packet algorithm}

\subsection{Wavelet processing of contaminant insulator flashover acoustic signal}

Wavelet analysis theory and the development of Fu Liye's theory have brought about a new era in methods of frequency analysis and tools for function approximation. Analysis of the contaminant insulator flashover acoustic signal can be the second part of the high and low frequencies using wavelet packet. Therefore, the wavelet packet analysis is a complete analysis process, and the resolution of the signal is more able to reflect the whole signal $[17,18]$.

The basic idea for wavelet packet decomposition of contaminant insulator flashover acoustic signal is to analyze sound signals' multi-resolution and decompose wavelet subspace. Typically by scaling function $\psi$ and wavelet $\phi$, the two decomposed parts are the low and high frequency acoustic signals, and approximation coefficients about acoustic signals sequence space is decomposed using a pair of filters. The relationships between scaling function, wavelet and filter are

$$
\begin{gathered}
\psi(t)=\sqrt{2} \sum_{k} h(k) \psi(2 t-k), \\
\phi(t)=\sqrt{2} \sum_{k} g(k) \phi(2 t-k), \\
U_{j}^{0}=U_{j+1}^{0} \oplus U_{j+1}^{1}, \quad j \in Z .
\end{gathered}
$$

According to the relation function space, Equations (1) and (2) reflect that the relationship is the same as (3).

In Equation (3), $U_{j}^{0}$ is the low-frequency part of the signal decomposition; $U_{j}^{1}$ is the high frequency part of the acoustic sound signal; $U_{j}^{1}$ is the orthogonal complement of $U_{j}^{0}$ and $U_{j-1}^{0} ; U_{j}^{0}$ is scale subspace; $U_{j}{ }^{1}$ is wavelet subspace; obviously $U_{j}^{0}$ and $U_{j}^{1}$ satisfy next formula:

$$
U_{j}^{0} \oplus U_{j}^{1} \oplus U_{j-1}^{1} \oplus U_{j-2}^{1} \ldots \oplus U_{j-m}^{1}=U_{j-m}^{0} .
$$

The space with different scale factors $j$, is all broken down into orthogonal subspace, $U_{j}{ }^{1}$ is the wavelet subspace of wavelet function $\psi(t)$ [18]. Gradually, wavelet subspace is divided into multiple frequencies, aimed to improve the acoustic signal frequency resolution.

By multiple resolution analysis, scaling function spanned subspace is $\left\{U_{j}^{0}\right\}$, and wavelet function spanned subspace is $\left\{U_{j}^{1}\right\}$. Closure spaces defined function is $u_{n}(t)$, and space closure of the function is subspace expansion coefficient. Subspace basis function is orthogonal subspace expansion coefficient groups and make the two-scale function satisfy the following Equations (5) and (6) [17, 18]. Subspace 
$U_{j}^{n}$ is closure space of function $u_{n}(t)$, subspace $U_{j}^{2 n}$ is closure space of function $u_{2 n}(t)$, expansion coefficient of basis function of subspace is $h(k)$, expansion coefficient of orthogonal basis of subspace is $g(k)$, to make function $u_{n}(t)$ satisfy the following two-scale equations:

$$
\begin{gathered}
u_{2 n}(t)=\sqrt{2} \sum_{k} h(k) u_{n}(2 t-k), \\
u_{2 n+1}(t)=\sqrt{2} \sum_{k} g(k) u_{n}(2 t-k) .
\end{gathered}
$$

When $n=0$, next equations can be directly derived:

$$
\begin{aligned}
& u_{0}(t)=\sqrt{2} \sum_{k} h(k) u_{0}(2 t-k), \\
& u_{1}(t)=\sqrt{2} \sum_{k} g(k) u_{0}(2 t-k) .
\end{aligned}
$$

By comparing (7) and (8) with the acoustic signal wavelet packet decomposition, subroutines $\phi(t)$ and $\psi(t)$, function $u_{1}(t)$ and function $u_{0}(t)$ are degenerated into wavelet function $\phi(t)$ and scaling function $\psi(t)$, and the two types of expression should be consistent. The promotion of this equivalence relation to the case $n \in Z^{+}$, sequences $\left\{u_{n}(t)\right\}_{n \in Z^{+}}$can be constructed.

The wavelet packet decomposition algorithm and the reconstruction algorithm are as follows:

Establish $g_{j}^{n}(t) \in U_{j}^{n}$, it can be represented as

$$
g_{j}^{n}(t)=\sum_{l} d_{l}^{j, n} u_{n}\left(2^{j} t-l\right) .
$$

Wavelet packet decomposition algorithm, uses $\left\{d_{l}^{j+1, n}\right\}$, to $\left\{d_{l}^{j, 2 n}\right\}$ and $\left\{d_{l}^{j, 2 n+1}\right\}$, namely:

$$
d_{l}^{j, 2 n}=\sum_{k} a_{k-2 l} d_{k}^{j+1, n}, d_{l}^{j, 2 n+1}=\sum_{k} b_{k-2 l} d_{k}^{j+1, n} .
$$

Wavelet packet reconstruction algorithm uses $\left\{d_{l}^{j+1,2 n}\right\}$ and $\left\{d_{l}^{j, 2 n+1}\right\}$ to $\left\{d_{l}^{j+1, n}\right\}$, namely:

$$
d_{l}^{j+1, n}=\sum_{k}\left[h_{l-2 k} d_{k}^{j, 2 n}+g_{l-2 k} d_{k}^{j, 2 n+1}\right] .
$$

Subspace $U_{j}^{n}$ is closure space of the function $u_{n}(t)$.

\subsection{Acoustic signal feature extraction of insulator based on wavelet packet analysis}

The above analysis reveals that the traditional wavelet packet decomposition algorithm can easily realize the decomposition and reconstruction. But the reconstruction of the signal analysis is not always needed, such as the signal feature extraction often needs only signal decomposition. Wavelet packet decomposition is to overcome the frequency of wavelet analysis in high frequency bands, as well as the defects of low resolution. However, the results of wavelet packet decomposition are characteristics in all frequency band of the signal; there is a lack of frequency selection and operation time is long. The results of the analysis take bigger number of storage units and are not conducive to real-time signal processing. Because the 
computation and decomposition levels are directly proportional, not all the band must be paid attention to. Usually only some band signal characteristics must be paid attention to, while some frequency bands of the information are not important. This paper uses a selective algorithm, and combines with the coefficient of dichotomy improved wavelet packet algorithm.

\subsubsection{General procedure of wavelet packets}

First, the sampled signal is $j$ layer wavelet packet decomposition respectively to extract the first $j$ layer from low frequency to high frequency of signal characteristics of all frequency components. The three layers of wavelet packet decomposition are shown on Fig. 4.

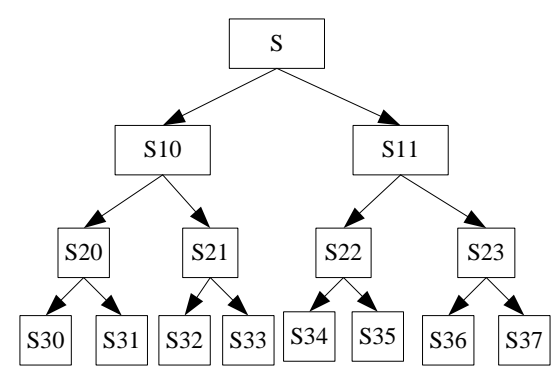

Fig. 4. Decomposition structure of three layers wavelet packet

Signal characteristics are manifested on node $(i, j)$ and the $j$-th node of the $i$-th layer. The original signal $S$ is $(0,0)$ node; $(1,0)$ represents $S 10$ the low frequency coefficient of the first layer of wavelet packet decomposition; $(1,1)$ represents S11 the high frequency coefficient of the first layer of wavelet packet decomposition, and so on.

According to the characteristics of the wavelet packet, the insulator flashover discharge sound signal design is decomposed into following steps:

Firstly, acoustic flashover signals $\mathrm{S}$ are captured, processed and decomposed into three levels by wavelet packet.

Secondly, the acoustic signal is reconstructed as

$$
\mathrm{S}=\mathrm{S} 30+\mathrm{S} 31+\mathrm{S} 32+\mathrm{S} 33+\mathrm{S} 34+\mathrm{S} 35+\mathrm{S} 36+\mathrm{S} 37 \text {. }
$$

Since the frequency of the insulator flashover discharge acoustic signals is within the range $0-60 \mathrm{kHz}$, the frequency range is shown in Table 2.

Table 2. Range of frequencies represented by the reconstructed signal

\begin{tabular}{|c|c|c|c|}
\hline $\begin{array}{c}\text { Reconstructed } \\
\text { signal }\end{array}$ & $\begin{array}{c}\text { Frequency range } \\
(\mathrm{kHz})\end{array}$ & $\begin{array}{c}\text { Reconstructed } \\
\text { signal }\end{array}$ & $\begin{array}{c}\text { Frequency range } \\
(\mathrm{kHz})\end{array}$ \\
\hline S30 & $0-7.5$ & S34 & $30-37.5$ \\
S31 & $7.5-15$ & S35 & $37.5-45$ \\
S32 & $15-22.5$ & S36 & $45-52.5$ \\
S33 & $22.5-30$ & S37 & $52.5-60$ \\
\hline
\end{tabular}

Thirdly, the energy $E_{j}, j=0, \ldots, 7$, of the reconstructed signal is calculated. Because the input signal is a random signal, the output signal is also a random 
signal, where $E_{j}=\sqrt{\sum_{k=1}^{N}\left|S_{3 j}(k)\right|^{2}}, S_{3 j}(k)$ is amplitude, $N$ is reconstruction signal points.

Finally, feature vectors are constructed. Insulator pollution flashover discharge will affect the signal energy of each frequency band. So, energy can construct a feature vector. Feature amount $F$ is expressed in:

$$
F=\left[\frac{E_{0}}{E}+\frac{E_{1}}{E}+\frac{E_{2}}{E}+\frac{E_{3}}{E}+\frac{E_{4}}{E}+\frac{E_{5}}{E}+\frac{E_{6}}{E}+\frac{E_{7}}{E}\right] .
$$

Among them, the total energy is $E=\sqrt{\sum_{j=0}^{7} E_{j}^{2}}$.

3.2.2. Energy analysis of contaminant insulator flashover acoustic signal on wavelet packet decomposition
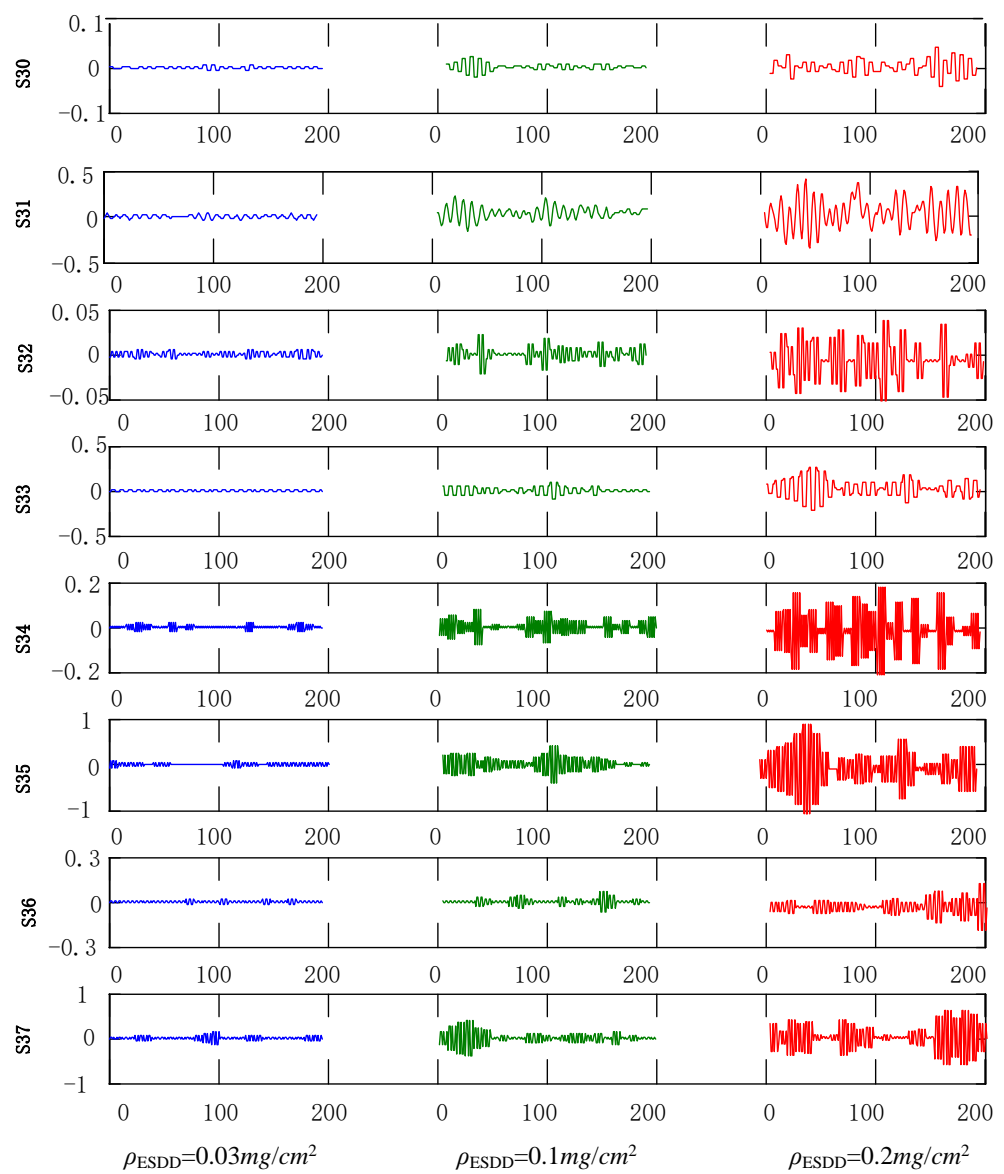

Fig. 5. The third layer reconstruction signal of wavelet packet decomposition of acoustic signal under three different ESDDs 
According to the step of decomposition, the $200 \mathrm{~ms}$ acoustic signal of this continuous discharge ESDD of the contaminant insulator flashover are decomposed by three layers of wavelet packet, and the energy characteristics are extracted of the frequency bands. In the experiment, the $3 \mathrm{~dB}$ wavelet is selected, which is a good tool to transform discrete wavelet. The wavelet packet decomposes the third layer coefficients to reconstruct the signal, as shown on Fig. 5, respectively $\rho_{\mathrm{ESDD}}=0.03 \mathrm{mg} / \mathrm{cm}^{2} ; \quad \rho_{\mathrm{ESDD}}=0.1 \mathrm{mg} / \mathrm{cm}^{2} ; \quad \rho_{\mathrm{ESDD}}=0.2 \mathrm{mg} / \mathrm{cm}^{2}$. The spectral characteristics of the reconstructed signal is shown on Fig. 6, where, for the purpose of simplicity and clarity, we plot $\rho_{\mathrm{ESDD}}=0.1 \mathrm{mg} / \mathrm{cm}^{2}$ and $\rho_{\mathrm{ESDD}}=0.2 \mathrm{mg} / \mathrm{cm}^{2}$.

Fig. 5 clearly shows that the main aspect to focus on are S31, S33, S35, S37 signals, which are all the high-frequency portions of the signal decomposition, after decomposing the contaminant insulator flashover acoustic signal by wavelet packet with three different test insulators' ESDDs.

Fig. 6 shows the spectral characteristics of the signal of different layers that have been decomposed. The power spectrum of S30, S32, S34 and S36 is weak, and the power spectrum of S31, S33, S35 and S37 is stronger than that of S30, S32, S34, S36, which is consistent with the results shown on Fig. 5. The energy of the S31 and S33 spectra is concentrated in $10-15 \mathrm{kHz}$, and the energy of the S35 and S37 spectra is concentrated in $35-40 \mathrm{kHz}$. Obviously the three ESDDs of the energy have a large difference with the increasing trend in the development of the flashing process of presented spectra.

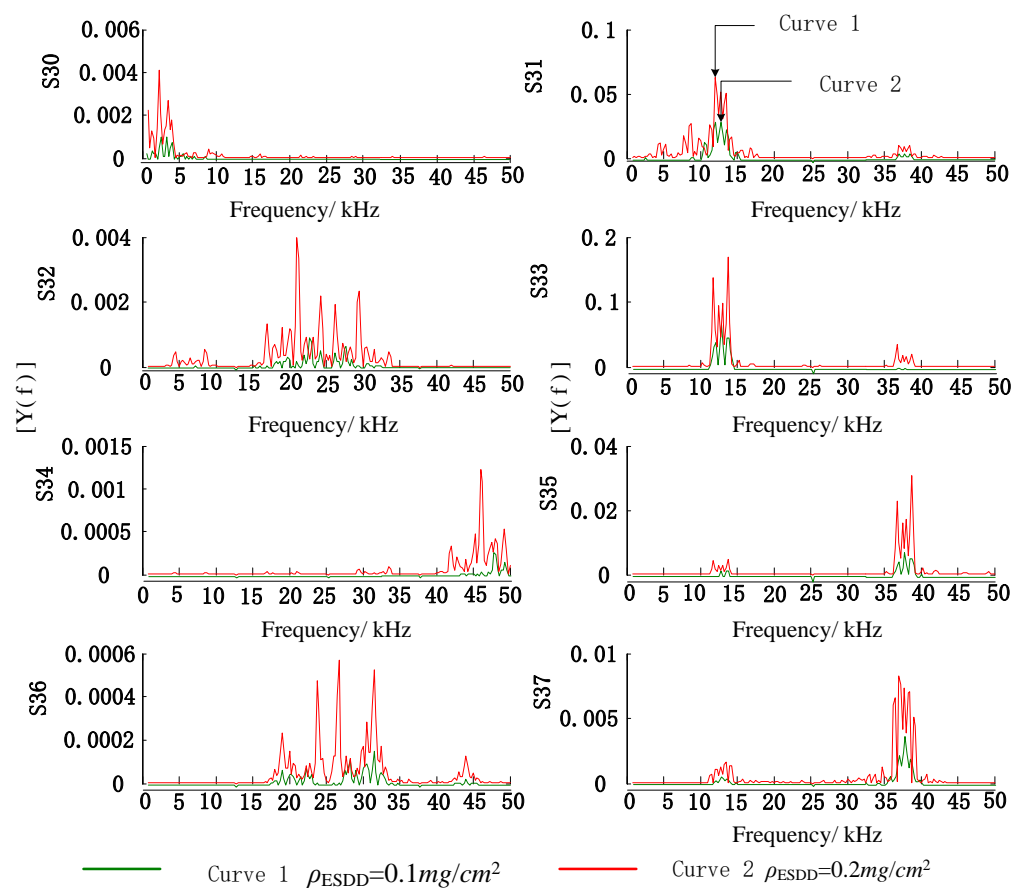

Fig. 6. Spectrum characteristics of two different ESDD three layers wavelet packet decomposition reconstruction signal 


\section{Conclusion}

This paper has studied the wavelet packet decomposition algorithm in contaminant insulator flashover acoustic signal analysis. Based on the spectral characteristics of wavelet packet acoustic signals, three layers wavelet packet decomposition of high frequency components was obtained. Fig. 6 shows that the three-layer-wavelet decomposition of high frequency components proportion is very high, and each node of decomposed high frequency component can be used as the basis for judging external insulation. Although the high frequency component of S31 and S33 is higher, the spectrum is between $10-15 \mathrm{kHz}$. The spectrum is formed by audible sound frequencies which are difficult to distinguish from audible noise and nature, and is prone to miscarriage. S35 and S37 frequency band energy is focused on $35-40 \mathrm{kHz}$, which are ultrasonic frequencies, and the frequency band energy is the occurrence of contaminant insulator flashover. So, S35, S37 can characterize contaminant insulator flashover characteristics, and can realize a good outer insulation waste state evaluation of the insulator.

Through the above analysis, four conclusions are drawn:

1) Contaminant insulator flashover from the three ESDDs of the acoustic signal has a significant difference. When the acoustic signal amplitude increases, the discharge signal pulse frequency increases.

2) The acoustic signal can reflect whether the contaminant insulator flashover is according to the variation of acoustic signals to judge the development trend of contaminant insulator flashover. Thus, the insulator contamination discharge monitoring and early warning can be realized.

3) The wavelet packet decomposition algorithm can be applied to the contaminant insulator flashover acoustic signal and a better decomposition result can be obtained.

4) The frequency signal in the third layer of wavelet packet during contaminant insulator flashover acoustic signal (especially S35 and S37) can be used as a basis for judging the flashover warning.

\section{References}

1. W a n g, F., K. Y u a n, N. S h u. Recognition of Contamination Discharge Development Stage of Insulators Based on Principal Component and Wavelet. - Insulating Materials, Vol. 48, 2015, No 4, pp. 52-56.

2. Qiangfeng, X. Research Status of Electrical Characteristics of Icing Post Insulator. Insulating Materials, Vol. 44, 2011, No 4, pp. 47-56.

3. Ranachowski, P., F. Rejmund, Z. Ran achowski et al. Acoustic Investigations of Long-Rod Insulators and Their Material Properties. - IEEE Transactions on Dielectrics and Electrical Insulation, Vol. 17, 2010, No 1, pp. 81-88.

4. Shao, C., Y. Zhen, T. Shu ang shuang. Study on Focusing Acoustic Sensor Device for Insulator Contamination Discharge Monitoring Method. - Electrical Measurement \& Instrumentation, Vol. 52, 2015, No 18, pp. 10-15.

5. Li, H., X. W e n, N. S h u et al. Application of Acoustic Emission Technology on Monitoring of Polluted Insulator Discharge. - In: Asia-Pacific Power and Energy Engineering Conference, (APPEEC'09), Wuhan, China, 2009, pp. 1-4. 
6. L u, M., Z. W a n g, X. Ji a n g. Application of the Ultrasonic Technology Based on Focused Sensor in Detecting Insulator Contamination Discharge. - East China Electric Power, Vol. 41, 2013, No 7, pp. 1569-1572.

7. W a n g, C., D. D a i, Y. Y a n g. Experimental Research on Acoustic Emission of Discharge from the Power Transmission System. - Automation of Electric Power Systems, Vol. 31, 2007, No 19, pp. 80-84.

8. W a n g, C. Research on Spreading Characteristics of Acoustics Emission Wave from Discharge of Insulators. - High Voltage Apparatus, Vol. 43, 2007, No 1, pp. 52-55.

9. W a n g, C. Experimental Research on Acoustics Emission from Discharge of Insulators. - High Voltage Apparatus, Vol. 32, 2006, No 3, pp. 51-53.

10. W a n g, C. Experiental Research on Acoustics Flashover Emission from Discharge of Insulators. In: Conference of the Power System Chinese Colleges and Automation Twenty Fourth Annual, 2008, pp. 1326-1329.

11. Li, T. Research on Acoustic Signal and Insulator Contamination Degree Prediction. - Shanxi Electric Power, 2010, No 3, pp. 38-41.

12. Li, H., X. W e n. Statistical Fingerprint Analysis for Contaminated Insulator Acoustic Emission Signals. - High Voltage Apparatus, Vol. 36, 2010, No 11, pp. 2705-2709.

13. Z h a n g, Z. Present Situation and Prospect of Research on Flashover Characteristics of Polluted Insulators. - Power System Technology, Vol. 30, 2006, No 2, pp. 35-40.

14. Li, L., L. T e n g, C. H u a n g. Envelope Analysis and Defects Identification of Partial Discharge U HF Signals in GIS. - High Voltage Engineering, Vol. 35, 2009, No 2, pp. 260-265.

15. S i, W., J. Li, P. Y u a n. Detection and Identification Techniques for Multi-PD Source in GIS. Proceedings of the CSEE, Vol. 29, 2009, No 16, pp. 119-126.

16. Q i, P. R. Porcelain Insulator Structure, Type of Effect on the AC Pollution Flashover Characteristics of the Research Status and Prospect. - Yunnan Electric Power Equipment Manufacturing Industry Revitalization and Innovation BBS, 2007, No 12, pp. 181-188.

17. Z h a n g, D. Matlab Wavelet Analysis. Mechanical Industry Press, 2009.

18. X u e, N. Matlab in the Application of Digital Signal Processing. Tsinghua University Press, 2003. 